\title{
Endodontic retreatment
}

\author{
Diksha Sinhaa, ${ }^{1, *}$, Dildeep Bali², Vijaya Dhar ${ }^{3}$, Era Arora $^{4}$, Suhrab Singh $^{5}$ \\ ${ }^{1}$ Post Graduate Student, ${ }^{2}$ Professor and HOD, ${ }^{3}$ Professor, ${ }^{4}$ Senior Lecturer, Dept. of Conservative Dentistry and Endodontics, \\ Santosh Dental College \& Hospital, Ghaziabad, Uttar Pradesh, India
}

Corresponding Author:

Email: dikshasinha2329@gmail.com

\begin{abstract}
Root canal treatment can last for lifetime, but in case of any recurrent infection and procedural errors endodontic retreatment can save a tooth. Whenever possible it is best to save a natural tooth. In some cases even when treatment has followed the highest standards it still results in endodontic failure. Endodontic failure results from persistent or secondary intra-radicular infection. Extra-radicular infections may be implicated in the failure of some cases. The reason many teeth do not respond to root canal treatment is because of procedural errors. The purpose of this article is to discuss about etiological factors of endodontic failure, removal of gutta percha from canals and different techniques of endodontic retreatment.
\end{abstract}

Keywords: Radicular infections, Overfilling, Coronal Leakage, Gutta percha, Retreatment, Endodontic failure, Curved canals, Rotary Instruments.

\section{Introduction}

Root canal treatments usually fail when treatment is inadequately done. Teeth with root canal treatment can last for a lifetime. A tooth that has been treated does not heal properly or remain diseased for months and years after treatment, if it develops new problems then a retreatment is required. The reason why a tooth does not respond to a root canal treatment is procedural errors and the persistence of microbial infection in the root canal system or the peri-radicular area.

There are several reasons associated with a tooth that may not heal as expected after initial treatment:-

1. Narrow or curved canals were not treated during the initial procedure.

2. Complicated canal anatomy went undetected in the first procedure.

3. Placement of the crown or other restoration delayed following the endodontic treatment.

4. Restoration did not prevent salivary contamination to the inside of the tooth.

5. New decay can expose the root canal filling material to bacteria, causing a new infection in the tooth.

6. Fractured tooth.

Some factors include microbial factors, comprising extra-radicular and intra-radicular infections, and intrinsic or extrinsic non-microbial factors. ${ }^{1}$

\section{Microbial Factors}

Intra-radicular Infection: In the root canal system microorganisms play an essential role in the pathogenesis of peri-radicular lesions. The dental pulps of conventional and germ free rats to oral cavity and reported that pulp necrosis and peri-radicular lesions developed only in conventional rats with an oral microbiota. $^{2}$ It was confirmed the important role of bacteria in peri-radicular lesions in a study using human teeth, in which bacteria were only found in root canals of pulpless teeth with peri-radicular bone destruction. The outcome of a root canal treatment are higher if infection is eradicated effectively before the root canal system is obturated. However the risk of reinfection will be dependent on the quality of the root filling and the coronal seal. ${ }^{3}$

Extra-radicular Infection: Peri-radicular lesions development creates a barrier within the body to prevent further spread of microorganisms. ${ }^{4}$ The resorption in bone tissue is seen and it is substituted by a granulomatous tissue containing defense elements such as cells (phagocytes) and molecules (antibodies and complement molecules). Polymorphonuclear leucocytes are composed of dense wall and an epithelial plug, is usually present at the apical foramen, blocking the egress of microorganisms in to the peri-radicular tissues. Cultural and microscopic studies have reported the occurrence of extra-radicular infections in both treated and untreated root canals. ${ }^{5}$

\section{Microbial Involvement in Special Situations}

Overfilling: The success rate of root canal treatment is decreased in cases of overfilling. Most of the obturating materials of a root canal system are biocompatible or show cytotoxicity only prior to setting. Over filling should be prevented since undesirable postoperative complications such as flare ups can develop. When a large amount of filling material extrudes through the apical foramen. The concomitant infection is an actual cause of failure of overfilled root canals emphasizes the need to properly prevent and control endodontic infection. $^{6}$

Coronal Sealing: Coronal leakage may be an important cause of an endodontic failure. In some situations obturated root canals may be contaminated from the oral cavity: leakage through the temporary / permanent restorative material, fracture of tooth structure, recurrent decay exposing the root canal filling or delay 
in placement of permanent restoration. ${ }^{7}$ The recontamination through coronal leakage causes sealer dissolution by saliva; percolation of saliva in the interface between sealer and gutta percha. ${ }^{6}$

Non Microbial Factors: In these cases, no microorganisms can be found, and failures have been attributed to a foreign body reaction in the periradicular tissues. It has been theorized that the formation of the cyst cavity is originated from immunological reactions, which attack epithelium in proliferation. $^{8}$

Instruments used for removal of gutta percha in cases of endodontic retreatment

The clinician can use various options for the removal of obturating material:

1. $\mathrm{K}$-files or $\mathrm{H}$-files

2. Gutta-percha solvent

3. Combination of paper points and gutta-percha solvent

4. Rotary instruments

a. Gates Glidden drill / Peesoreamers

b. GPX gutta-percha remover

c. Ni Ti rotary instruments

5. Specialized rotary instruments designed for retreatment

a. Pro Taper Universal retreatment instruments

b. Mtwo retreatment files

c. R-Endo retreatment files

6. Heat transfer devices

a. Heat carrier tips

b. Ultrasonic tips

7. Soft tissue laser.

$\mathrm{Ni}$ Ti rotary files are very effective and efficient for removing gutta percha. $\mathrm{Ni} \mathrm{Ti}$ instruments are used for shaping canals at a speed of approximately 300 RPM. For rotary removal of gutta percha. ProTaper Retreatment instruments (D1-D3, Dentsply Tulsa Dental) at approximately 500-750 RPM as greater rotational speed is necessary to create the friction and heat necessary to thermosoften gutta percha. ${ }^{8}$ The blades of these nickel titanium instruments engage softened gutta percha and effectively takes out this material out of a root canal space. Certainly, hand files in the presence of a chemical, such as chloroform, is another important method to remove gutta percha from smaller and more curved canals. Chloroform rapidly softens gutta percha and, in conjunction with files, allows for the removal of gutta percha in a crown-down manner. With the canals filled with chloroform bath then paper points are utilized to wick residual gutta percha and sealers from the more inaccessible regions of the root canal system. ${ }^{9}$ The removal method has been coronal $1 / 3$, middle $1 / 3$, apical $1 / 3$; eliminating gutta percha in a crown-down manner. The Pro Taper Retreatment series (D1-D3, Dentsply Tulsa Dental) work safely and efficiently in this manner. This goes a long ways towards preventing the possibility of pushing gutta percha through the foramen, whether you're dealing with heat- or chemically-softened gutta percha. Removing gutta percha requires higher RPM's than for cleaning and shaping procedures. The recommended speed is around 500-750 RPM. The bottom line is whatever speed creates enough friction that mechanically softens and takes out gutta percha is fine. ${ }^{10}$

Removing all gutta percha from the root canal prior to commencing with cleaning and shaping procedures. If a NiTi rotary file's taper is too large to accomplish this goal, then use smaller tapered NiTi rotary instruments first. In more underprepared canals, or anatomically challenging canals, use 0.02 tapered stainless steel hand files in the presence of a solvent such as chloroform. Chloroform is perhaps a little more effective than xylol in the removal of gutta percha and residual sealers. It works faster and more efficiently if you use it carefully. ${ }^{10}$

An important method to remove gutta percha, especially when the canal has been overextended vertically and under filled laterally, is to utilize the hedstroem displacement technique. The gutta percha is first thermosoftened with heat and then a 35,40 , or 45 hedstroem file is passively rotated clockwise into this mass. Let the gutta percha cool and harden within the blades, and upon withdrawing the hedstroem file, oftentimes the entire mass of gutta percha will be removed as well.

In many instances a silver point extends out of the canal and into the pulp chamber. Clinicians need to thoughtfully perform access so as not to inadvertently foreshorten a silver point which could result in making it more difficult to grasp and pull out. It's important to remove all circumferential material surrounding the silver point to facilitate loosening and removal. A grasping tool such as the Stieglitz pliers (Henry Schein) can generally get a strong purchase on the coronal end of a silver point and then, utilizing the concept of fulcrum mechanics, elevate the silver point out of the canal. $^{9}$

Indirect ultrasonics is another important method to remove silver points. It is not wise to place any ultrasonic instrument directly on the silver point because it will rapidly erode away this soft material. Rather, first engage the silver point with a grasping instrument and then place an ultrasonic instrument, such as a ProUltra Endo Tip \#1 (Dentsply Tulsa Dental), on the pliers to indirectly vibrate it loose. Since most canals are irregular in their cross-sectional dimensions and since silver points are round, then theoretically, space exists between the wall of the canal and the silver point. Files, solvents, and chelators can be utilized to eliminate sealer thus undermining and loosening the silver point so it can be removed.

The hedstroem displacement technique is an invaluable technique for removing silver points or silver point segments. Finally, microtubes can be utilized to engage and remove certain silver points. The 
Post Removal System (PRS, SybronEndo) has small microtubular taps, allowing the clinician to mechanically tap and engage any silver point that is 0.6 $\mathrm{mm}$ or greater in diameter and coronal extent extends into the pulp chamber. The successful removal of carrier-based obturators utilizes the same techniques for removing gutta percha and silver points. Oftentimes, the biggest secret to remove a carrier is patience and perseverance. When evaluating a paste case for retreatment, it is wise to recognize that certain pastes are very difficult to remove because they set up brick hard. However, it is important to appreciate that due to

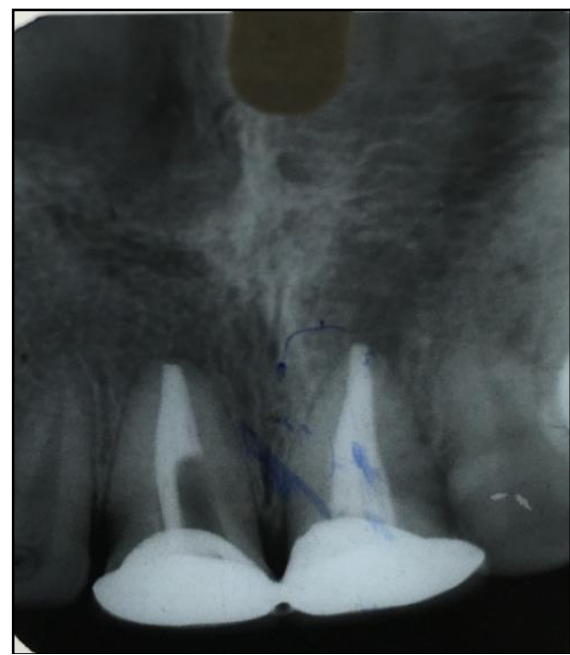

Fig. 1: Pre-operative IOPA gutta percha removal

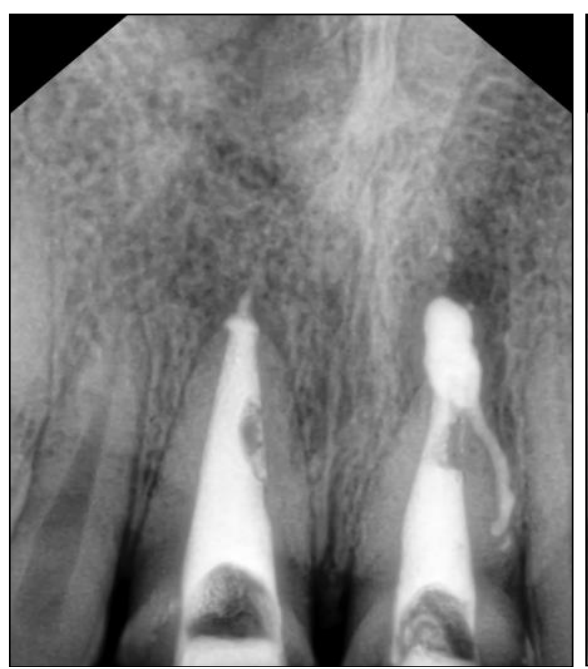

Fig. 2: Metapex obturation

Techniques of Endodontic Retreatment: For removal of gutta-percha as well as the new resin reinforced gutta-percha one can use heat and solvents (i.e, chloroform), LAAxxes burs (Sybron Endo, Orange, CA), Gates-Glidden drills, and nickel titanium rotary files at a higher RPM (ie, 1200 RPM). With guttapercha carrier systems such as Thermafil (Dentsply, Tulsa Dental, York, PA) in addition to the above the method of placement, the most dense portion of the paste is in the coronal one-third and the material is generally less dense moving in an apical direction. To remove paste apical to canal curvature, use a pre-curved stainless steel file attached to a specially designed "File Adapter" (Sybron Endo) that hooks to the ultrasonic hand piece (P5, Dentsply Tulsa Dental). Other removal methods include heat, end-cutting rotary NiTi instruments, chemicals such as Endosolv $\mathrm{R}$ and Endosolv E (Endoco) and Micro-Debriders (Dentsply Maillefer). ${ }^{8}$
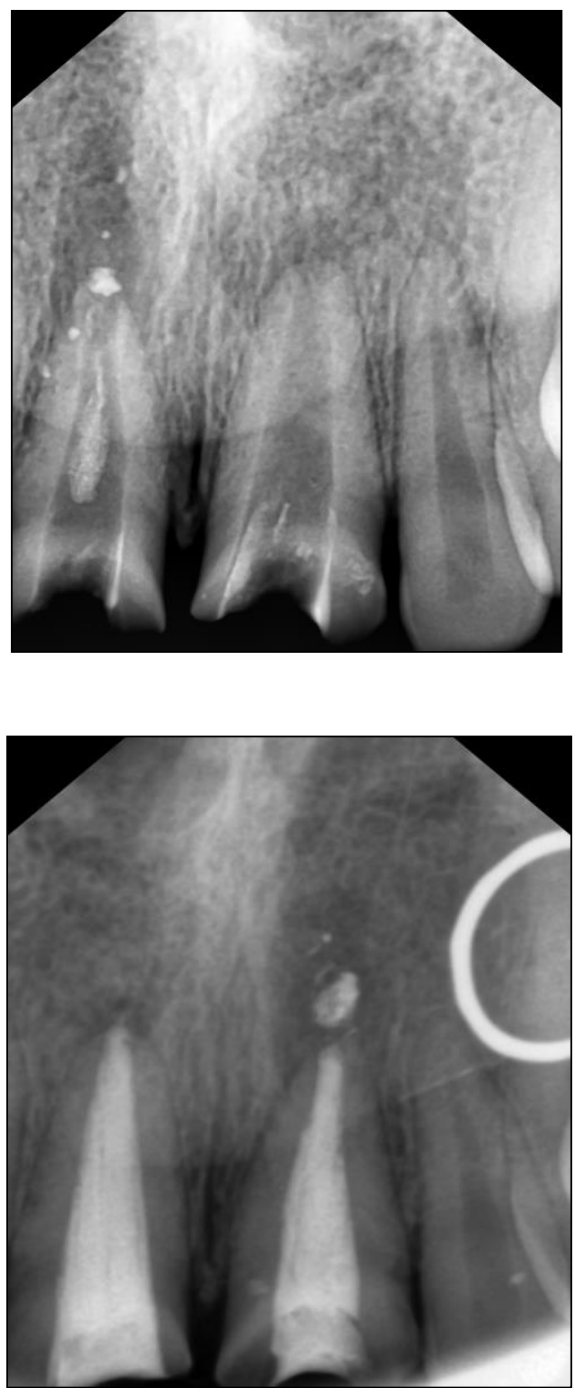

mentioned mechanisms, one can also use the braiding technique, where the gutta-percha is bypassed on two sides and then two or more Hedström files are threaded past the Thermafil carrier, twisting the handles of the two Hedströms to remove the gutta percha/Thermafil as a unit. Once the gutta-percha has been removed it is important to utilize an apex locator to determine the "new" working length. There are often periapical radiolucencies associated with teeth requiring 
retreatment and the radiographic apex will not be the same as the actual clinical apex, especially when resorption is present. ${ }^{8}$

Therefore, the use of an apex locator is critical for determining the apical extent of the obturation. Retreatments often require the removal of metal posts, pins, silver points or other obstructions such as separated instruments. There are several types of forceps available, such as Steiglitz, Peete, Hemostats, etc. and you can make your own instrument by cutting a groove into the tip of a spoon excavator, giving the appearance of a snake' stongue.

This can be extremely helpful in elevating out a pin once it has been loosened up with ultrasonics. The first step in removal of posts/pins etc. is the use of diamond coated ultrasonic tips (Spartan Corp., Fenton, MO). They are used at a low frequency setting without water.

A fine stream of air is blown through airway syringe, which removes excess debris and reduces the heat generated by these ultrasonic tips. They are designed to efficiently remove the cement around a post/pin helping to loosen it up. They are not designed to purposefully vibrate directly against the metal obstruction. Another instrument helpful in loosening a post is a Rotopro (Ellman Corp, Port Washington, NY). These are non-cutting fluted stainless steel instruments used with water in a high-speed hand piece. By going around the post in a counterclockwise direction the cement will chip away and the threads of the post will start to unwind. By utilizing the above-mentioned techniques, we can more predictably and efficiently perform endodontic retreatments. ${ }^{10}$

\section{Case Reports in Department of Conservative Dentistry and Endodontics Santosh Dental College and Hospital}

Case 1: A female patient was reported with the chief complaint of pain and swelling in upper front region. On examination it was reported that the root canal treatment was done 2 years back. Radiograph findings, gutta percha was extruded beyond the apical foramen and periapical infection was seen. After the removal of gutta percha metapex dressing was given. Here are the radiograph images showing pre-operative and postoperative conditions.
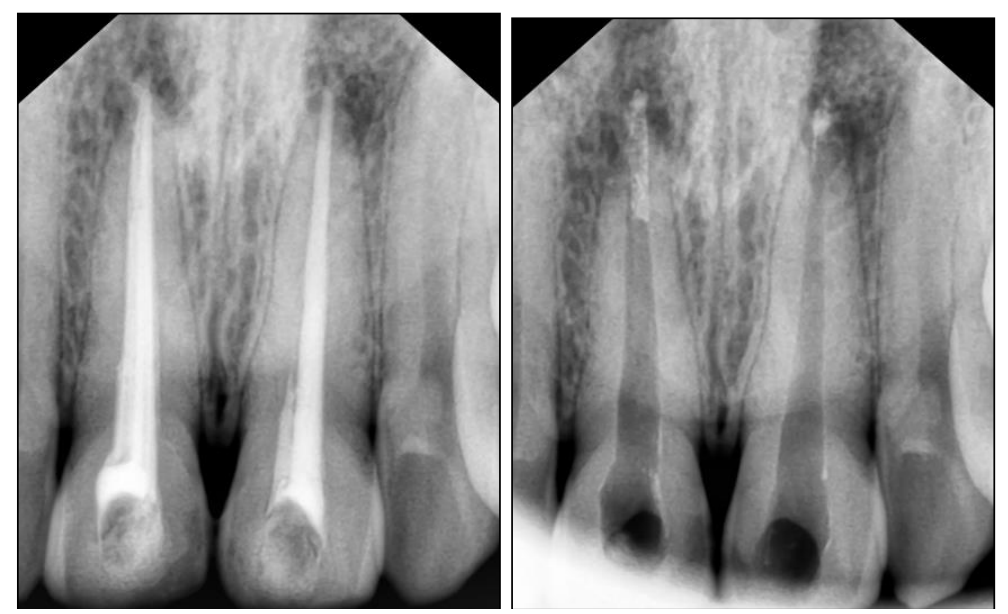

Fig. 3: Pre-operative IOPA gutta percha removal
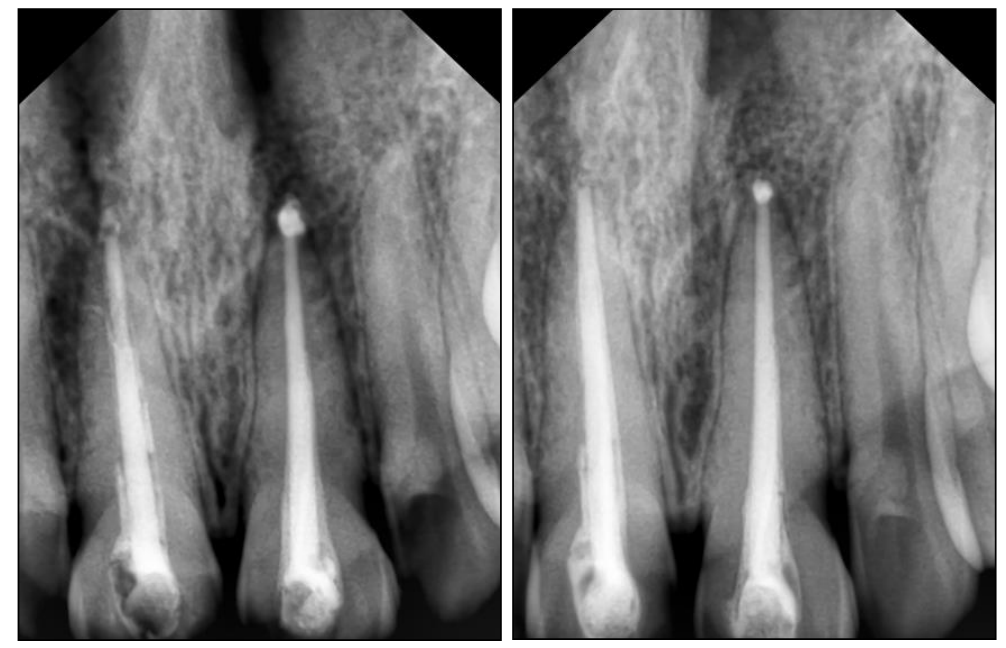

Fig. 4: Metapex obturation 
Case 2: A male patient was reported with the chief complaint of pain in the upper back region. On examination it was reported that root canal treatment was done 5 years back. Radiograph findings, gutta

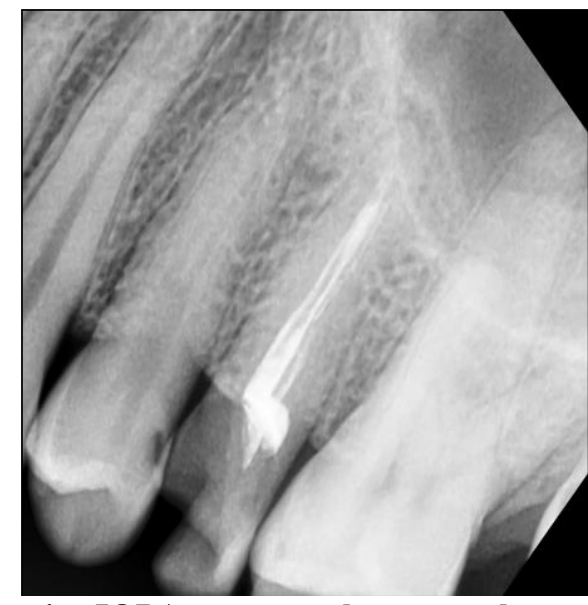

Fig. 5: Pre-operative IOPA gutta percha removal

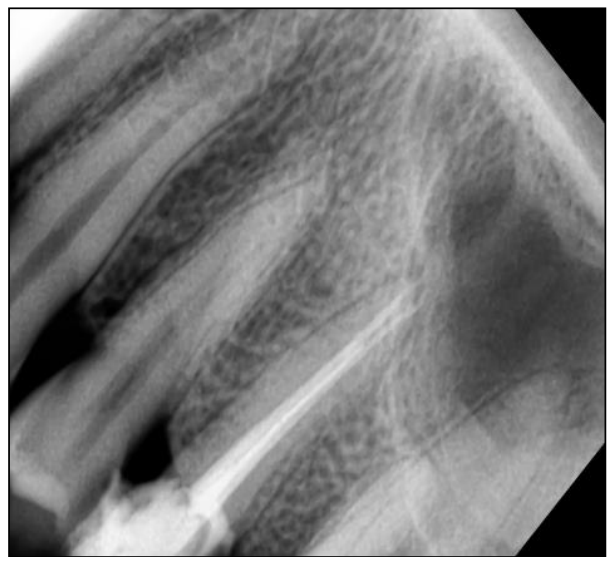

Fig. 6: Obturation

\section{Conclusion}

Endodontic retreatment should be done with the utmost care, it is important to save a natural tooth. Advances in technology are constantly changing the way root canal treatment is performed, so endodontic retreatment may be able to resolve the problems. As with any dental or medical procedure, we always discuss options and the chances of success before beginning retreatment.

\section{References}

1. Siquiera J. F. Jr. Etiology of root canal treatment failure: why well-treated teeth can fail. Int Endod J 2001;34(1): 110.

2. Removal of Obturation Materials- Advanced Endodontics 6/30/16. Available from:

http://www.endoruddle.com/FAQRetxObtur

3. Samuel kratchman, Frank C Setzer. Endodontic Retreatment Techniques. USA ENDO TRIBUNE U.S Edition.

4. Samuel kratchman, Mian Iqbal. Endodontic Retreatment Techniques. 2006;2:1-9. percha was short in length and instrumentation of canals were inadequately done. Here are the radiograph images showing pre-operative and post-operative conditions.

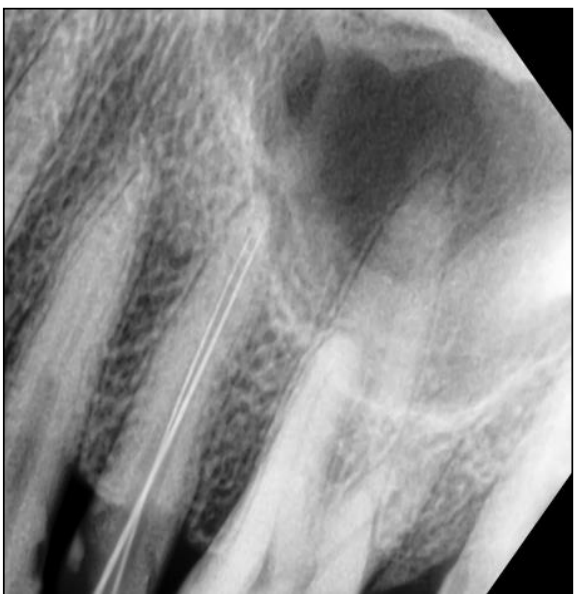

5. Gu LS, Ling JQ, Wei X, Huang XY. Efficacy of Pro taper Universal rotary retreatment system for gutta-percha removal from root canals. Int Endod J 2008;41(4):28895.

6. Emre Bodrumlu, Ozgur Uzun, Ozgur Topuz, Mustafa Semiz. Efficacy of 3 techniques in removing Root Canal Filling Material. J Can Dent Asso 2008;74(8):721a-721d.

7. Neslihan Simsek, Ali Keles, Fuat Ahmetoglu, Mevlut Sinan Ocak, Saim Yologlu. Comparison of different retreatment techniques and root canal sealers: a scanning electron microscopic study. Braz Oral res 2014;28(1):111.

8. A step by step explanation of endodontic retreatment by American association of endodontists 6/30/16. Available from : http://www.aae.org/patients/treatments-and procedures/endodontic-retreatment-explained.aspx

9. Roheet Khatavkar, Vivek Hegde. Current concepts in gutta-percha removal for re-treatment, Dental Tribune, April-June 2010. Available from: https://www.dentaltribune.com/epaper/dt-india-archived/dt-india-archived0210-[18-18].pdf

10. Instruments and techniques used in Removal of Gutta percha Colgate Oral Care October 10,2013. Available from: http://www.juniordentist.com/instruments-andtechniques-used-in-removal-of-gutta-percha.html 